\title{
Evidence supporting platform-switching to preserve marginal bone levels not definitive
}

\author{
Abstracted from \\ Atieh MA, Ibrahim HM, Atieh AH. \\ Platform switching for marginal bone preservation around dental implants: \\ a systematic review and meta-analysis. J Periodontol 2010; 81: 1350-1366. \\ Address for correspondence: Sir John Walsh Research Institute, School of Dentistry, \\ University of Otago, Dunedin, New Zealand. E-mail: maatieh@gmail.com
}

\section{Question: Does platform switching preserve alveolar bone?}

Data sources MEDLINE, EMBASE, The Cochrane Oral Health Group's Trials Register, The Cochrane Central Register of Controlled Trials (CENTRAL), UK National Research Register, Australian New Zealand Clinical Trials Registry (ANZCTR), Database of Abstracts of Reviews of Effectiveness (DARE), ISI Proceedings for relevant conference abstracts. The search strategy used keywords but not subject heading terms. A number of relevant journals were hand searched (seven most recent years) and authors were contacted in the absence of complete data. Study selection Randomised controlled trials (RCT) or controlled clinical trials (CCT) reported in English only, that compared platformswitched to platform-matched implants were eligible. A minimum of 10 implants had to have been placed in the platform-switched group (it is unclear if there was a minimum for the comparison group) and they had to have been followed up for a minimum of 12 months. Primary outcome was marginal bone level changes. Secondary outcome was implant failure rate.

Data extraction and synthesis Data were extracted by more than one author using a data extraction form. Quality assessment was done using the Jadad scale. Meta-analysis was conducted using fixed effects model in the absence of significant heterogeneity, and the random effects model where heterogeneity was greater. Statistical heterogeneity was assessed using the chi ${ }^{2}$ and $\mathrm{I}^{2}$ tests. Sensitivity and subgroup analyses were planned to identify any potential causes of heterogeneity. Results Ten studies including 1239 implants were included and all were published 2007-2010. Seven were RCTs, three were CCTs. Range of observation was 12-60 months. Methodological quality was assessed as 'satisfactory'. Chi ${ }^{2}=126.79(\mathrm{P}<0.0001), \mathrm{I}^{2}=91 \%$ indicating significant statistical heterogeneity. Thus the random effects model was used to synthesise the data. Bone loss in the platform-matched implant group was greater with a mean difference of $-0.37 \mathrm{~mm}(95 \% \mathrm{Cl}-0.55$ to $-0.20, \mathrm{P}<0.0001)$. This is based on the longest follow-up interval from each trial (therefore could be anywhere between 12 and 60 months). There was no significant difference in implant failure. Conclusions Platform-switching may preserve vertical crestal bone levels more than platform-matching when placing implants.

\section{Commentary}

Marginal bone loss around implant fixtures after surgical placement and loading is well studied and documented in the literature, with radiographic bone loss ranges of $1.5 \mathrm{~mm}$ during the first year, followed by $0.2 \mathrm{~mm}$ in subsequent years being an important parameter in assessing the success of the implant fixture. ${ }^{1}$

In recent years, platform-switching has been increasingly investigated as a viable technique to decrease the amount of the marginal bone loss that occurs around an implant collar when it is exposed to the oral environment. Platform-switching involves the placement of a smaller diameter prosthetic component on a larger diameter implant fixture. This connection shifts the perimeter of the implant-abutment junction (IAJ) inward towards the central axis of the implant. ${ }^{2}$ The rationale is that shifting the IAJ inward also repositions the inflammatory cell infiltrate and confines it within a $90^{\circ}$ area, thereby reducing the amount of marginal bone loss; a concept first theorised by Lazzara and Porter. ${ }^{3}$

The authors' stated aim for this systematic review and meta-analysis was to investigate whether or not there was an evidence-based rationale for the use of platform-switched, as opposed to platformmatched components in the preservation of marginal bone levels. A secondary, but no less important outcome of implant failure rates when using platform-switching, was evaluated.

The ten eligible studies chosen were all English language studies published between the years 2007-2010, utilising human participants that directly compared platform-switched vs. platform-matched implants in either Randomised Control Trials (RCTs) or Controlled Clinical Trials (CCTs). Only one of the ten eligible studies included in this systematic review and meta-analysis was a long-term prospective study (CCT - 60 months) with the other nine varying in length from 12 months (3 RCTs, 1 CCT), to 24 months (2 RCTs, 1 CCT), to 27 months (1 RCT) and finally 33 months (1 RCT).

A major strength of this systematic review and meta-analysis was in the authors' use of the most recent guidelines of PRISMA (Preferred Reporting Items for Systematic Reviews and MetaAnalyses) ${ }^{4}$ and the Cochrane Collaboration methods ${ }^{5}$ to evaluate the best available evidence for the use of platform-switching as a design feature to limit peri-implant bone loss around implants. The PRISMA guidelines were developed to help authors improve the reporting of systematic reviews and meta-analyses and adopted the definitions used by the Cochrane Collaboration. When used as a basis for reporting, the PRISMA methodology helps to ensure a more consistent, higher quality outcome. By the authors' use of 


\section{RESTORATIVE DENTISTRY}

this methodology in conducting their systematic review and subsequent meta-analysis, the reader can be assured not only that the appropriate amount of due diligence was performed, but that it was also done in a logical, prescribed manner. The authors' use of a welldefined and focused PICO question that helped to summarise their objectives and inclusion criteria, and which also acted as an aid in their evidence-based search of the literature, is laudable.

Potential drawbacks to this study were noted by the authors, and they specifically mentioned the limitation of using conventional radiographs to assess buccal and lingual bone levels, as well as noting that mesial and distal bone levels were assessed in only one dimension; vertically. Although radiographs remain one of the most convenient and readily accessible diagnostic methods to evaluate crestal bone loss, they do have limitations. Radiographs clearly represent the mesial and distal aspect of the implant, but they fail to show accurately the facial/buccal aspect where bone loss often occurs. ${ }^{6}$ The authors did a good job of recognising and discussing the limitations of their review while making compelling defenses in their study design, approach and results.

A more recent systematic review that included seven of the ten articles in this systematic review and meta-analysis would seem to corroborate the authors' conclusions. ${ }^{7}$ The authors of this publication were unable to perform a meta-analysis due to concern with the heterogeneity among the included publications in terms of surgical protocols (submerged vs. non-submerged and crestal vs. subcrestal placement), loading protocols (immediate vs. delayed), and platform surface configuration (smooth vs. threaded), but were able to provide narrative detail on the outcomes of the selected articles. They also concluded that platform-switching seemed to have some beneficial effect on peri-implant marginal bone levels.

With only one long-term study available, the evidence supporting the use of platform-switching to preserve marginal bone levels

\section{Practice points}

- The current evidence supporting the use of platform-switching is not definitive, however the rationale behind platform-switching and the potential benefits from using this technique make it an attractive option.

- With no statistically significant difference in implant fixture failure rates between platform-switched and platform-matched components, the clinician can maintain the option of selecting either technique with no adverse patient effects.

is certainly not definitive, but the results from this meta-analysis as well as other, more recent studies assert that the inward shift of the IAJ is a desirable morphological feature that may preserve vertical crestal bone levels.

Gary L Stafford

Department of General Dental Sciences, Marquette University School of Dentistry, Milwaukee, Wisconsin, USA

1. Albrektsson T, Zarb G, Worthington P, Eriksson AR. The long-term efficacy of currently used dental implants: a review and proposed criteria of success. Int J Oral Maxillofac Implants 1986; 1: 11-25.

2. Prasad DK, Shetty M, Bansal N, Hegde C. Crestal bone preservation: a review of different approaches for successful implant therapy. Indian I Dent Res 2011; 22: 317-323.

3. Lazzara RJ, Porter SS. Platform switching: a new concept in implant dentistry for controlling postrestorative crestal bone levels. Int J Periodontics Restorative Dent 2006; 26: 9-17.

4. Liberati A, Altman DG, Tetzlaff |, et al. The PRISMA statement for reporting systematic reviews and meta-analyses of studies that evaluate healthcare interventions: explanation and elaboration. Br Med / 2009; 339: b2700 doi: 10.1136/bmj.b2700

5. Higgins ], Green, $\mathrm{S}$ (eds). Cochrane handbook for systematic reviews of interventions. Wiley 2011

6. Misch, CE. Dental Implant Prosthetics. 1e. Mosby; 2004

7. Al-Nsour MM, Chan HL, Wang HL. Effect of the platform-switching technique on preservation of peri-implant marginal bone: a systematic review. Int J Oral Maxillofac Implants 2012; 27: 138-145.

Evidence-Based Dentistry (2012) 13, 56-57. doi:10.1038/sj.ebd.6400864 